\title{
Using host-pathogen protein interactions to identify and characterize Francisella tularensis virulence factors
}

\author{
Anders Wallqvist', Vesna Memišević ${ }^{1}$, Nela Zavaljevski ${ }^{1}$, Rembert Pieper ${ }^{2}$, Seesandra V. Rajagopala²,
} Keehwan Kwon'², Chenggang Yu', Timothy A. Hoover ${ }^{3}$ and Jaques Reifman ${ }^{1 *}$

\begin{abstract}
Background: Francisella tularensis is a select bio-threat agent and one of the most virulent intracellular pathogens known, requiring just a few organisms to establish an infection. Although several virulence factors are known, we lack an understanding of virulence factors that act through host-pathogen protein interactions to promote infection. To address these issues in the highly infectious F. tularensis subsp. tularensis Schu S4 strain, we deployed a combined in silico, in vitro, and in vivo analysis to identify virulence factors and their interactions with host proteins to characterize bacterial infection mechanisms.
\end{abstract}

Results: We initially used comparative genomics and literature to identify and select a set of 49 putative and known virulence factors for analysis. Each protein was then subjected to proteome-scale yeast two-hybrid (Y2H) screens with human and murine cDNA libraries to identify potential host-pathogen protein-protein interactions. Based on the bacterial protein interaction profile with both hosts, we selected seven novel putative virulence factors for mutant construction and animal validation experiments. We were able to create five transposon insertion mutants and used them in an intranasal BALB/c mouse challenge model to establish $50 \%$ lethal dose estimates. Three of these, $\triangle \mathrm{FTT} 0482 \mathrm{C}, \Delta \mathrm{FTT1538c}$, and $\Delta \mathrm{FTT1597}$, showed attenuation in lethality and can thus be considered novel $F$. tularensis virulence factors. The analysis of the accompanying $\mathrm{Y} 2 \mathrm{H}$ data identified intracellular protein trafficking between the early endosome to the late endosome as an important component in virulence attenuation for these virulence factors. Furthermore, we also used the $\mathrm{Y} 2 \mathrm{H}$ data to investigate host protein binding of two known virulence factors, showing that direct protein binding was a component in the modulation of the inflammatory response via activation of mitogen-activated protein kinases and in the oxidative stress response.

Conclusions: Direct interactions with specific host proteins and the ability to influence interactions among host proteins are important components for F. tularensis to avoid host-cell defense mechanisms and successfully establish an infection. Although direct host-pathogen protein-protein binding is only one aspect of Francisella virulence, it is a critical component in directly manipulating and interfering with cellular processes in the host cell.

Keywords: Host-pathogen interactions, Protein-protein interactions, Francisella tularensis, Intracellular pathogen, Virulence factors

\footnotetext{
* Correspondence: jaques.reifman.civ@mail.mil

'Department of Defense Biotechnology High Performance Computing

Software Applications Institute, Telemedicine and Advanced Technology

Research Center, U.S. Army Medical Research and Materiel Command, Fort

Detrick, MD 21702, USA

Full list of author information is available at the end of the article
} 


\section{Background}

Francisella tularensis, the causative agent of tularemia and a Centers for Disease Control and Prevention Category A select agent, is one of the most infectious bacteria known, with $<10$ cells capable of causing severe infections in humans and animals [1]. Its role as a biological weapon has been investigated in the United States (U.S.), the former Soviet Union, and Japan [2], and it remains both a potential health hazard and a bioterrorism threat. F. tularensis is a Gram-negative opportunistic intracellular pathogen that primarily affects lagomorphs but can infect virtually any human cell type, although the primary infection typically goes through macrophages $[3,4]$. As such, F. tularensis is endowed with multiple robust infection mechanisms, including the ability to avoid or circumvent host immune defenses [5-11].

Relatively little is known about the detailed molecular nature of $F$. tularensis pathogenicity, although recent progress in high-throughput genomic and proteomic technologies has contributed to an increase in the number of experimentally confirmed or predicted genes involved in its virulence [12]. By definition, inactivation of these "virulence factors" attenuates or abrogates virulence in an animal infection model, regardless of which stages of the infection process they are involved in. Given the high virulence and large number of identified virulence factors for this organism, one can surmise that multiple robust mechanisms are contributing to the infection process.

Francisella spp. virulence is highly correlated with the presence of the so-called pathogenicity islands among the most pathogenic strains and their modification or absence in non-pathogenic strains. A 33-kbp pathogenicity island has been identified in F. tularensis [13], and systematic mutations confirmed its key role in virulence [14]. A region of the pathogenicity island encodes a set of genes that map to a fully functional type VI secretion system (T6SS) $[15,16]$. In F. tularensis, T6SSs play a central role in manipulating host cells by translocating virulence factors across the bacterium's inner and outer membranes directly into the host cytosol. Alternatively, bacterial proteins can also be exported to the periplasmic space via the universal Sec pathway and then translocated across the outer membrane, usually via a type II secretion system (T2SS) [17, 18]. The identity of the specific proteins translocated through these systems and their interactions with host proteins or other factors in the host cell are not well characterized. However, host-pathogen interactions provide a means to identify virulence factors that are directly related to protein interactions and ascertain their associated mechanisms of infectivity $[19,20]$.

Accordingly, we deployed a bioinformatics-based approach to initially select $F$. tularensis subsp. tularensis
Schu S4 proteins broadly associated with secretion and filtered the selected putative virulence factors through whole-genome human and murine yeast two-hybrid $(\mathrm{Y} 2 \mathrm{H})$ screens to select unstudied bacterial proteins that exhibited host-pathogen protein-protein interactions (PPIs). Seven of these bacterial proteins were selected for transposon mutant construction, five of which were successfully obtained and used in an intranasal BALB/c mouse challenge model to ascertain in vivo virulence. Three of these, $\triangle$ FTT0482c, $\triangle$ FTT1538c, and $\Delta$ FTT1597, showed a statistically significant reduction in lethality compared with the wild-type strain and can, thus, be considered novel virulence factors. Based on the accompanying $\mathrm{Y} 2 \mathrm{H}$ data, we could also generate hypotheses about the underlying host mechanisms targeted by infecting bacteria.

\section{Methods}

Bioinformatics and literature-based identification of potential virulence factor proteins

We initially identified and collected putative Francisella spp. virulence factor proteins based on comparative genomics between high- and low-pathogenicity strains, the presence of $\mathrm{VgrG}$ domains as indicative of T6SS association [21], model predictions of signaling sequences, and literature-based searches. We obtained all Francisella genomes from the PathoSystems Resource Integration Center database [22].

Using QuartetS [23, 24], we performed whole-genome comparisons of six highly pathogenic $F$. tularensis strains with eight less pathogenic $F$. tularensis strains and seven F. novicida strains (Additional file 1: Table S1) to identify proteins present only in the highly pathogenic strains. To identify putative VgrG proteins, we initially downloaded VgrG domain-containing genes from the National Center for Biotechnology Information (NCBI) database [25]. Using BLAST [26], we compared the $F$. tularensis subsp. tularensis Schu S4 genome with all other VgrG domain-containing genes and identified putative $F$. tularensis VgrG genes based on homology. We considered two genes as homologs if the E-value of their alignment was $\leq 0.01$.

We used SignalP to predict the presence and location of signal peptide cleavage sites in amino acid sequences of the F. tularensis subsp. tularensis Schu S4 genome [27]. In prokaryotes, these signal peptides constitute ubiquitous protein-sorting signals that target their passenger proteins for translocation across the cytoplasmic membrane, although the presence of the signal peptide does not guarantee that a protein is secreted. We used both the hidden Markov model method and the neural network method in SignalP to identify $F$. tularensis proteins with signal peptides to tag them as putatively secreted proteins. We used default cutoff values for both 
methods and designated putative signaling proteins as those proteins identified using both methods.

\section{High-throughput $\mathrm{Y} 2 \mathrm{H}$ screens to identify human- $F$. tularensis PPIs}

We cloned the known and predicted F. tularensis virulence factor genes from $F$. tularensis subsp. tularensis Schu S4 genomic DNA. All F. tularensis virulence factor genes were PCR amplified using gene-specific primers incorporated with forward and reverse Gateway recombination cloning sequences, i.e., attB1 (5'-GGGGAC AAGTTTGTACAAAAAAGCAGGCTTC-3') and attB2 (5'-GGGGACCACTTTGTACAAGAAAGCTGGGTC-3'), respectively. The PCR-amplified open reading frames (ORFs) were cloned into Gateway entry vector pDONR221 ${ }^{\mathrm{sm}}$ as recommended by the BP Clonase ${ }^{\mathrm{mit}}$ II enzyme provider (Invitrogen, Carlsbad, CA). We validated the entry vectors with the cloned ORFs by Sanger sequencing.

Entry clones were sub-cloned into $\mathrm{Y} 2 \mathrm{H}$ bait (DNAbinding domain) vectors, pGBGT7g (as $\mathrm{NH}_{2}$-terminal fusion) and pGBACg (C-terminal fusion) [28], using Gateway LR reactions (Invitrogen). Y2H bait clones were subsequently transferred into the haploid yeast strain AH109 (MAT- $\alpha$ ), as previously described [29]. Before the two-hybrid analyses, we examined all baits in yeast strains for auto-activation, i.e., detectable bait-dependent reporter gene activation in the absence of any interacting protein. We used the HIS3 reporter gene, and autoactivation was titrated by adding different concentrations of 3-amino-1,2,4-triazole (3-AT), a competitive inhibitor of HIS3. All $F$. tularensis baits were inspected for autoactivation on plates containing different concentrations of 3-AT. The lowest concentration of 3-AT that suppressed growth for the interaction screen was used because it avoided background growth while still detecting true interactions.

A haploid yeast strain expressing each $F$. tularensis protein as bait was used for the PPI screening with human and mouse normalized universal cDNA libraries (catalog nos. 630480 and 630482, Clontech Laboratories, Mountain View, CA). The bait and prey yeast culture was grown and mixed at a 1:1 ratio and plated on yeast extract, peptone, dextrose, and adenine (YEPDA) agar plates. YEPDA agar plates were incubated at $30{ }^{\circ} \mathrm{C}$ for $6 \mathrm{~h}$ or overnight at room temperature. During this process, both prey and bait plasmids were combined in diploid yeast cells by yeast mating. Cells from the mating plates were collected and transferred onto interactionselection yeast-synthetic medium with predefined concentrations of 3-AT (media lacking tryptophan, leucine, and histidine plus 3-AT), and plates were incubated at $30{ }^{\circ} \mathrm{C}$ for $4-6$ days. The interaction-selection plates that showed colony growth but no colonies on control plates (bait mated to empty prey vector) were identified as two-hybrid positive yeast clones. Positive yeast colonies were selected manually and subjected to yeast colony PCR followed by DNA sequencing to identify the interacting preys [29].

\section{Pairwise $\mathrm{Y} 2 \mathrm{H}$ retesting of human-F. tularensis high- throughput PPIs}

To increase confidence in selected human- $F$. tularensis virulence factor protein interactions, we designated 12 interaction pairs identified in the high-throughput $\mathrm{Y} 2 \mathrm{H}$ library screening for retesting in pairwise $\mathrm{Y} 2 \mathrm{H}$ assays. Human prey clones were constructed by sub-cloning the ORFs from the Human ORFeome collection [30] or amplifying the ORF from the Mammalian Gene Collection library [31] and subsequently transferred into the two $\mathrm{Y} 2 \mathrm{H}$ prey vectors (pGADT7g and pGBACg). The pairwise $\mathrm{Y} 2 \mathrm{H}$ assays were performed with activation tests conducted side by side as previously described [29].

\section{Generation of transposon insertion mutants}

The F. tularensis subsp. tularensis Schu S4 strain used in this study was derived from a U.S. Army Medical Research Institute for Infectious Diseases (USAMRIID) stock of an $F$. tularensis isolate obtained from a U.S. patient in 2005 and supplied by McKesson BioServices (Rockville, MD). A seed stock of that strain was passaged twice through mice, and single colony isolates were collected, pooled, and labeled FT12. In these experiments, in vitro culturing of FT12 was done at $37^{\circ} \mathrm{C}$ in enhanced tularemia broth (ETB) consisting of trypticase soy broth (Becton Dickinson, Franklin Lakes, NJ) supplemented with cysteine $(0.1 \%)$, glucose $(0.1 \%)$, ferric pyrophosphate $(0.25 \%)$, and horse serum (2.5\%). Growth of FT12 on solid medium was done on enhanced tularemia agar (ETA) plates consisting of Mueller-Hinton II agar (Becton Dickinson) supplemented with $\mathrm{NaCl}(2.5 \mathrm{~g} / \mathrm{l})$, protease peptone $(5 \mathrm{~g} / \mathrm{l})$, cysteine $(0.1 \%)$, glucose $(0.1 \%)$, ferric pyrophosphate $(0.25 \%)$, and horse serum $(2.5 \%)$.

Plasmid pGreenhopper (T.A. Hoover, unpublished data) was constructed in plasmid pCR2.1-TOPO (Life Technologies, Frederick, MD) and carries a modified Mariner-based Himar 1 transposon and flanking transposase gene. Within the inverted DNA repeats that define the termini of the transposon is a kanamycin resistance gene positioned downstream of the promoter for the FT12 groESL operon to allow for selection of cells harboring transposon insertions. pGreenhopper was electroporated into FT12, diluted, and plated on 20 ETA plates. Approximately 2,000 colonies per plate were scraped and pooled for use in high-throughput mutagenesis screening experiments. Southern blot analysis of 30 independent $F$. tularensis transposon recipients suggested that the library was random and that transposition events occurred once per cell, presumably due to the lack of 
replication of pGreenhopper in F. tularensis and the conservative cut-and-paste mode of replication characteristic of Mariner family transposable elements.

Specific F. tularensis Schu S4 mutants were isolated from the pooled collection of approximately 40,000 independent transposon insertion mutants by nested PCR detection with progressively smaller library subsets. Primers that annealed downstream of a gene of interest were paired with primers that annealed to the terminal repeats of the inserted transposon in PCRs containing small aliquots of whole cells from subsets of the F. tularensis library (Table 1). Subsets giving rise to appropriately sized DNA bands from these reactions were cultured on ETA plates to obtain smaller subsets, and the process was repeated until single colony isolates were obtained and stored at $-80{ }^{\circ} \mathrm{C}$. Genomic DNAs were prepared from these clones and re-analyzed by PCR to confirm the presence of the transposon within the coding sequence of each gene. In two cases (FTT1538c and FTT1564), insertion sites were determined by DNA sequencing.

\section{Animal experimentation}

$\mathrm{BALB} / \mathrm{c}$ mice have been widely used and accepted for the experimental study of tularemia and was the animal model chosen for these experiments. Female BALB/c mice at 6-9 wk of age were obtained and intranasally administered doses of wild-type F. tularensis Schu S4 and selected mutants, and then observed for 21 days. Moribund mice and mice surviving to day 21 post-challenge were euthanized in accordance with USAMRIID Standard Operating Procedure AC-11-04-05.

Challenge doses were prepared from overnight $F$. tularensis cultures grown at $37^{\circ} \mathrm{C}$ on ETA plates. Cells were scraped and diluted in phosphate-buffered saline solution to obtain appropriate numbers of cells in each dose. Intranasal installations of $33 \mu \mathrm{l} /$ mouse were followed by $10-\mu \mathrm{l}$ washes in each nostril. Mice were

Table 1 Primers, plasmids, and strains used in the mouse intranasal challenge model experiments

\begin{tabular}{|c|c|c|}
\hline Primer, plasmid, or strain & Relevant characteristics & Source or reference \\
\hline \multicolumn{3}{|l|}{ Primer } \\
\hline FTT0482d5' & GCTCAACATTGTATGAAATTAATGGCTCCA & Invitrogen \\
\hline FTT0482p5' & GTGAAATAGTCAGACAAGTAAGCCTTGGT & Invitrogen \\
\hline FTT0482d3' & ATTTGTATCAGCCAAATGCTGTTACGCA & Invitrogen \\
\hline FTT0482p3' & GTAGAATGTGGATGAATGTTAAGTACGGT & Invitrogen \\
\hline FTT0902d5' & CTGCTGCTGCTCAGACAGCTACTACTG & Invitrogen \\
\hline FTT0902p5' & GCTGCTGCTGTATCTAAGCCAACTGC & Invitrogen \\
\hline FTT1538d5' & CAGCAGGCGATTATGGCTACAAACA & Invitrogen \\
\hline FTT1538p5' & GCAGGTCAGATGTCGACACAAGAAGC & Invitrogen \\
\hline FTT1538d3' & TGCTTCAGCTTCGGACTTAGCAACA & Invitrogen \\
\hline FTT1538p3' & ATCCAATTGCTGCATCCACACCATCA & Invitrogen \\
\hline FTT1564d5' & СTCCTCCTCATATCAGTTCTGTCAGCT & Invitrogen \\
\hline FTT1564p5' & GTCGGTITCCCAAGCTACTGGAATGT & Invitrogen \\
\hline $\mathrm{FTT1564d3^{ \prime }}$ & GGACTCGAACCTACGACCTACGGAT & Invitrogen \\
\hline FTT1564p3' & CTAACCAACTGAGCTATAGGCCCA & Invitrogen \\
\hline $\mathrm{FTT} 1597 \mathrm{~d} 5^{\prime}$ & CGGCTGATAATGATGGCTTTATGGCT & Invitrogen \\
\hline FTT1597p5' & TGATCCTCCTGAATATGATGATCCTAGT & Invitrogen \\
\hline FTT1597d3' & GCATATACGGCTGAATCTTGCCACCT & Invitrogen \\
\hline FTT1597p3' & CAATGATGCCAATGCCGCGGTAACT & Invitrogen \\
\hline \multicolumn{3}{|l|}{ Plasmid } \\
\hline pCR2.1-TOPO & 3,931-bp TA vector; pMB1 oriR; $\mathrm{Km}^{\mathrm{r}}$ & Life Technologies \\
\hline pGreenhopper & $\begin{array}{l}\text { pCR2.1-TOPO containing a 4-kbp Mariner-Himar } 1 \text { transposon with kanamycin } \\
\text { resistance gene and a } 1.2 \mathrm{kbp} \text { tnpA transposition gene. Non-replicable in F. tularensis }\end{array}$ & Unpublished \\
\hline \multicolumn{3}{|l|}{ E. coli } \\
\hline TOP10 & General cloning and blue/white screening & Life Technologies \\
\hline \multicolumn{3}{|l|}{ F. tularensis } \\
\hline FT12 & Type strain, a mouse-passaged isolate of a strain obtained from a U.S. patient & McKesson BioServices \\
\hline
\end{tabular}


anesthetized during this procedure by an intramuscular injection of $100 \mu \mathrm{l}$ of ketamine-acepromazine-xylazine in accordance with USAMRIID Standard Operating Procedure AC-09-10.

This study was conducted in compliance with the Animal Welfare Act and other federal statutes and regulations relating to animals and experiments involving animals and with adherence to principles stated in the Guide for the Care and Use of Laboratory Animals (www.nap.edu/readingroom/books/labrats/chaps.html).

The facility where this research was conducted is fully accredited by the Association for Assessment and Accreditation of Laboratory Animal Care International. The USAMRIID Institutional Animal Care and Use Committee reviewed and approved the animal protocol entitled "Pathogenesis studies of $F$. tularensis mutants in mice," (Animal Protocol no. AP-11-013).

\section{Growth rate experiments}

Select F. tularensis subsp. tularensis Schu S4 strain transposon insertion mutants were cultured in chemically defined Chamberlain's growth medium, and their growth rates, as determined by optical density measurements at $600 \mathrm{~nm}\left(\mathrm{OD}_{600}\right)$, were compared to those of the parent strain. Frozen stocks of each mutant and the parent strain were thawed on ice and diluted into $2 \mathrm{ml}$ growth media at an $\mathrm{OD}_{600}$ of $\sim 0.150$. Culture tubes were incubated at $37{ }^{\circ} \mathrm{C}$ while constrained in a slanted rack and shaken at $150 \mathrm{rpm}$. Cultures were sampled for $\mathrm{OD}_{600}$ readings as a function of time, and their values were recorded.

\section{Biosafety and biosecurity}

The Francisella experiments were conducted in Biosafety Level 3 containment laboratories at USAMRIID with appropriate personal safety and biological select agents and toxins security measures in place. We do not anticipate that this report provides knowledge, products, or technologies that could be directly misapplied by others to pose a threat to public health and safety, agricultural crops and other plants, animals, the environment, or materiel. The Institutional Biosafety Committee (IBC) that approved this work is composed of members of the USAMRIID research staff, Commander's office, and qualified representatives from external institutions and is tasked to provide local, institutional oversight of research using recombinant DNA. The USAMRIID IBC was established under the U.S. National Institutes of Health Guidelines for Research Involving Recombinant DNA Molecules.

\section{$50 \%$ Lethal dose estimation}

We used Bayesian probit analyses to estimate the lethal dose response. As a prior distribution, we used a weakly informative Cauchy distribution with center 0 and scale 10. Parameters were assumed independent of each other. Using samples from the posterior distribution and intercept parameters from the probit analysis, we estimated the $50 \%$ lethal dose $\left(\mathrm{LD}_{50}\right)$ and $95 \%$ confidence intervals as well as the likelihoods of each strain being more or less potent than any other strain at the median lethality level.

\section{Survival time comparison}

We used the Kaplan-Meier method to estimate the probability of animals surviving a given length of time [32] and a G-rho class of rank tests to compare difference between the obtained survival curves [33]. We calculated all probabilities using the $\mathrm{R}$ analysis package "survival" (Terry Therneau, "A Package for Survival Analysis in S," R package version 2.37-7, 2014).

Creation of the expanded human-F. tularensis PPI network We used the NCBI HomoloGene database of homologs (www.ncbi.nlm.nih.gov/homologene) to identify humanmurine orthologs [34]. These orthologs were further used to create sets of 1) conserved PPIs between human-F. tularensis and murine-F. tularensis and 2) expanded F. tularensis-human PPIs consisting of experimentally detected and orthology-based predicted protein interactions.

We identified the conserved set of PPIs as those PPIs where the human protein interacted with the same $F$. tularensis protein as their murine orthologs. To create the expanded set of $F$. tularensis-human PPIs we merged the human data with orthologous mouse data. To identify the orthologous data, we first identified the $11 \mathrm{~F}$. tularensis proteins that had protein interactions with both hosts. Among these interactions, we identified the set of murine-F. tularensis PPIs for which there exists a human ortholog to the murine protein. The resulting orthologous data set consisted of 76 interactions between 74 human proteins and the $11 F$. tularensis proteins, corresponding to $64 \%$ of the murine- $F$. tularensis PPIs. Finally, we merged the human- $F$. tularensis experimental and orthologous data sets to create the expanded set of human- $F$. tularensis PPIs consisting of 298 unique interactions between 18 F. tularensis and 249 human proteins. These PPI data sets are provided in the supplementary information (Additional file 2: PPI data).

All networks were plotted in Cytoscape [35] using $\mathrm{R}$ packages iGraph and RCytoscape [36, 37].

\section{Gene Ontology annotation of host genes}

We performed two types of Gene Ontology (GO) [38] enrichment analysis: standard enrichment analysis and network-based enrichment analysis. Both enrichment analyses were performed in $\mathrm{R}$ using the Bioconductor packages BioMart [39]. GO annotations were obtained from BioMart [39]. We used all annotation levels of the 
GO tree, excluding only terms associated with the root and top two levels. We used the Benjamini and Hochberg multiple test correction to account for multiple hypothesis testing [40].

In the standard enrichment analysis, we used the hypergeometric distribution to assess the statistical significance of observing a given GO term in our data. As the complement of human proteins, we used the set of all human/ murine proteins available in BioMart [39] and annotated with at least one $\mathrm{GO}$ term. The reported results contain only GO terms that were enriched at a $\leq 20 \%$ false discovery rate.

\section{Network analysis of host-pathogen PPIs: interaction modules}

In the network-based enrichment analysis, we first identified connected (sub)networks of the largest connected component (LCC) in the human interactome that consists of human proteins interacting with at least one F. tularensis protein and in which all human proteins were annotated with the same GO term. We denote these (sub)networks as "interaction modules." We then assessed the statistical significance of observing a given interaction module using Monte Carlo simulation. Specifically, we assessed probabilities of observing the same interaction module given 1) a set of random human proteins of the same size as the set of human proteins interacting with $F$. tularensis and 2) a set of random (randomly rewired) human PPIs, where the degree (number of interactions) of each protein was maintained to the same value as in the full set of human PPIs [20]. We kept only interaction modules consisting of four or more proteins. For the full set of human proteins, we used all constituent proteins from a human PPI network [41]. The reported results contain only GO terms that were enriched at a $\leq 5 \%$ false discovery rate.

\section{Results}

In silico identification of potential virulence factors

We used multiple independent strategies to identify potential virulence factors of $F$. tularensis subsp. tularensis Schu S4. We initially used orthology to identify eight proteins present only in six genomes of highly pathogenic $F$. tularensis genomes and absent in eight less pathogenic $F$. tularensis and seven $F$. novicida genomes (Additional file 1: Table S1) [23, 24]. Given the importance of T6SSs in Francisella virulence [21], we further identified 16 putative proteins with homology to VgrG domain-containing proteins and, thus, potentially a component of the T6SS. We used SignalP to identify 290 proteins predicted to be secreted by the general secretory pathway. Finally, we examined the available literature on Francisella high-throughput and small- scale virulence screens to compile a list of 300 proteins associated with virulence [3-6, 12, 42-44].

\section{Selection of potential virulence factor proteins for $\mathrm{Y} 2 \mathrm{H}$ experiments}

To select proteins for experimental evaluation, we first merged the lists of potential virulence factors and downselected proteins based on expert knowledge weighing the available evidence. As our focus was on detecting novel virulence factors, we initially excluded proteins previously studied in animal model experiments. From the list of predicted secreted proteins, we excluded 226 proteins 1) lacking annotations and predicted to be secreted with a probability lower than 1.0 or 2) proteins with annotations but predicted to be secreted with a probability of $<0.9$. From the list of 300 experimentally screened proteins, we excluded capsule proteins whose role in virulence is controversial [45], acid phosphatases as they have recently been proven less important for virulence [46], and metabolic proteins and bacterial regulators as these proteins are not likely to be secreted. We also excluded all membrane and transporter proteins except those that were identified from our comparative genomics or signal peptide analysis. Furthermore, we removed type IV pili proteins and proteins associated with Francisella pathogenicity islands encoding T6SS, as they have already been extensively studied in animal model experiments. The exceptions were type IV pili protein FTT1314c, predicted to be secreted by the general secretory pathway and putatively associated with the T2SS [47], and the T6SS proteins FTT1712c (IgIC2) and FTT1707 (IgIl2) that have been shown to be secreted in proteomics experiments $[15,48]$ and to contribute to pathogenicity $[14,16]$, but their host interacting partners are not known. Furthermore, we kept all proteins annotated as hypothetical or of unknown function, as long as their orthologs were present in the F. tularensis subsp. tularensis Schu S4 genome.

We further removed all proteins shorter than 90 amino acids and proteins annotated as essential in the Database of Essential Genes [49], as these latter proteins would prevent mutant creation. The resulting list of proteins contained 119 unique potential virulence factors. Of these, 97 had not previously been tested in murine models; 22 had been tested in murine models, but their interactions with host proteins are not known. We then ranked and grouped the set of 119 proteins into five groups based on the confidence level we could associate with each protein as a secreted virulence factor. We assigned proteins the highest level of confidence for those with multiple known virulence associations and evidence about attenuation in a mouse animal model from high-throughput screening of $F$. tularensis subsp. tularensis Schu S4 using the Transposon Directed 
Insertion Site Sequencing technique (T.A. Hoover, unpublished data). We assigned the lowest level of confidence to those proteins for which we only had orthology-based evidence.

Finally, we individually assessed each protein and down-selected the list into a final, pruned set of 49 putative and known virulence factors suitable for experimental evaluation (Table 2).

\section{$\mathrm{Y} 2 \mathrm{H}$ screening of host-pathogen interactions}

All 49 putative and known virulence factors were successfully cloned, prepared, and tested in $\mathrm{Y} 2 \mathrm{H}$ assays against both human and murine whole proteome libraries. Y2H screens identified 407 interactions between $F$. tularensis baits and human preys and 170 interactions between $F$. tularensis baits and murine preys. All baits were mapped to their corresponding $F$. tularensis locus tags, and all preys were mapped to their official gene symbols as defined in the HUGO Gene Nomenclature Committee database [50] or Mouse Genome Informatics database [51]. We were not able to map some of the identified preys to protein coding sequences, and interactions containing those preys were removed. Moreover, we removed protein interactions between $F$. tularensis and "sticky" host proteins known to be indiscriminate binders (F. Schwarz and M. Koegl, unpublished data). The final data consisted of 222 unique PPIs between $18 F$. tularensis and 183 human proteins and 118 unique PPIs between $13 F$. tularensis and 113 murine proteins (Fig. 1). Eleven F. tularensis proteins interacted with both hosts, seven interacted only with human proteins, and two interacted only with murine proteins. As shown in Fig. 1, the majority of F. tularensis proteins interacted with unique host proteins, i.e., 137 (75 \%) human proteins and 110 (97 \%) murine proteins interacted with a single $F$. tularensis protein. Furthermore, $\mathrm{Y} 2 \mathrm{H}$ screens identified 21 conserved PPIs between these two data sets, i.e., interactions in which human proteins interacted with the same $F$. tularensis proteins as their murine orthologs. These conserved interactions consisted of six F. tularensis proteins and 20 unique host proteins. Table 2 shows the number of unique PPIs between each tested $F$. tularensis protein and human and murine proteins as well as the corresponding number of conserved interactions in terms of orthologous pairs of interactions. The small overlap between $F$. tularensis-human and $F$. tularensis-murine PPIs was potentially due to low quantities of a prey gene in one of the two cDNA libraries or to nonexhaustive sampling of host-prey and pathogen-bait protein interactions. However, the experimental conditions were the same for both sets of experiments. Thus, our detected interactions represent a subset of all interactions that can occur.

\section{Transposon mutant selection and in vitro growth}

To identify a small set of $F$. tularensis protein candidates for validation in animal model experiments, we focused on nine proteins that interacted with multiple proteins in both hosts. From this set of proteins, we first removed two $F$. tularensis proteins that had already been tested for virulence attenuation in animal models: FTT0356 [44] and FTT0626 [43]. We then evaluated and ranked each of the remaining seven proteins based on the number of conserved interactions in which they participate or based on involvement of their host targets in biological processes and pathways related to virulence, e.g., signaling and apoptosis. This procedure resulted in seven previously uncharacterized virulence factor candidates: FTT0482c, FTT0842, FTT0902, FTT1029, FTT1538c, FTT1564, and FTT1597.

We successfully obtained from the transposon library insertion mutants in the F. tularensis subsp. tularensis Schu S4 strain for five virulence factor candidates (FTT0482c, FTT0902, FTT1538c, FTT1564, and FTT1597). We were unable to obtain an insertion mutant for the peptidoglycanassociated lipoprotein FTT0842 and FTT1029. Growth rate experiments in Chamberlain's chemically defined growth medium [52] during the exponential phase showed that there was no significant difference in the growth rate for the mutants compared with those of the parent strain except for $\triangle$ FTT1564, which showed a minor growth enhancement (Additional file 3: Table S2).

The created transposon mutants are potentially subject to polar effects. Based on available computational bioinformatics information in the DOOR [53] and ProOpDB [54] databases, FTT0482c and FTT1597 belong to annotated single-gene operons; thus, they should not be associated with polar effects. On the other hand, FTT1538c belongs to an operon of four genes (FTT1537c to FTT1540c) and polar effects are theoretically possible. Further studies are required to definitively determine possible polar effects.

\section{Virulence attenuation in a murine intranasal infection model}

Using intranasal infection, we gauged the effects of the five insertion mutations on $F$. tularensis virulence relative to the fully virulent wild-type $F$. tularensis subsp. tularensis Schu S4 strain. A total of 360 BALB/c mice [10 mice for each of six doses of increasing numbers of colony-forming units (CFUs) for each of the six strains (five mutant strains + the wild-type strain)] were exposed to different intranasal doses ranging from $\geq 0.03 \mathrm{CFU}$ to $\geq 78,000 \mathrm{CFU}$ and monitored for 21 days (Additional file 4: Table S3 and Additional file 5: Figure S1). We used these dose-response experiments to estimate the lethal dose for each strain and compare the potency of each strain. Estimated wild-type $\mathrm{LD}_{50}$ 
Table $\mathbf{2}$ List of proteins selected for experimental yeast two-hybrid evaluation

\begin{tabular}{|c|c|c|c|c|c|c|}
\hline \multirow[t]{2}{*}{ Group } & \multirow[t]{2}{*}{ Locus ID } & \multirow[t]{2}{*}{ Name } & \multirow[t]{2}{*}{ Description } & \multicolumn{3}{|c|}{ Interactions } \\
\hline & & & & Human & Mouse & Common \\
\hline \multirow[t]{15}{*}{1} & FTT0369c & DipA & Deficient in intracellular replication A & - & - & - \\
\hline & FTT0584 & - & Unknown & - & - & - \\
\hline & FTT0901 & LpnA & Lipoprotein & - & 1 & - \\
\hline & FTT0918 & FopC & Outer membrane lipoprotein & - & - & - \\
\hline & FTT1103 & DsbA & Conserved hypothetical lipoprotein & - & - & - \\
\hline & FTT1179 & BipA & GTP binding translational elongation factor Tu & 29 & - & - \\
\hline & FTT1508c & RelA & GTP pyrophosphokinase & 3 & - & - \\
\hline & FTT1726 & YegQ & Protease YegQ & - & - & - \\
\hline & FTT1676 & - & Protein of unknown function, membrane protein & - & - & - \\
\hline & FTT0356 & HtpG & Chaperone, heat shock protein $\mathrm{HtpG}$, ATPase activity & 20 & 1 & 1 \\
\hline & FTT0626 & Lon & Chaperone, ATP-dependent DNA-binding protease Lon & 2 & 2 & - \\
\hline & FTT1769c & ClpB & Chaperone, ATP-dependent CLP protease ATP-binding subunit ClpB & - & - & - \\
\hline & $\mathrm{FTT} 1712 \mathrm{C}$ & $\lg \mid \mathrm{C} 2$ & Intracellular growth locus, subunit $C$ & 43 & 33 & 1 \\
\hline & FTT0068 & SodB & Superoxide dismutase & - & 12 & - \\
\hline & FTT1707 & $\lg \mid 12$ & Uncharacterized protein & - & - & - \\
\hline \multirow[t]{16}{*}{2} & FTT0013c & - & Protein of unknown function & - & - & - \\
\hline & FTT0295 & - & Hypothetical protein & - & - & - \\
\hline & FTT0520 & - & Putative uncharacterized protein & - & - & - \\
\hline & FTT0842 & - & Peptidoglycan-associated lipoprotein & 9 & 17 & - \\
\hline & FTT0902 & - & Protein of unknown function & 8 & 1 & - \\
\hline & FTT0975 & - & Protein of unknown function & - & - & - \\
\hline & FTT1039 & DacB1 & D-Alanyl-D-alanine carboxypeptidase (penicillin-binding protein) family protein & - & - & - \\
\hline & FTT1040 & - & Lipoprotein & - & - & - \\
\hline & FTT1334C & - & Hypothetical protein & 1 & 1 & - \\
\hline & $\mathrm{FTT} 1402 \mathrm{C}$ & - & Protein of unknown function & - & - & - \\
\hline & FTT1538c & - & Hypothetical protein & 25 & 22 & - \\
\hline & FTT1549 & - & Protein of unknown function & - & - & - \\
\hline & FTT1626c & - & Hypothetical protein & - & - & - \\
\hline & FTT1688 & - & Aromatic amino acid HAAP transporter & - & - & - \\
\hline & FTT1314C & PilE6 & Type IV pili fiber building block protein & 4 & - & - \\
\hline & FTT1776c & - & Hypothetical protein & 2 & - & - \\
\hline \multirow[t]{6}{*}{3} & FTT0086 & - & Conserved protein of unknown function & - & - & - \\
\hline & FTT0103C & - & Hypothetical protein & 2 & - & - \\
\hline & FTT0792 & - & LPS locus & - & - & - \\
\hline & FTT0889c & - & Type IV pili fiber building block protein & - & - & - \\
\hline & FTT1530 & FadB/AcbP & Bifunctional 3-hydroxacyl-CoA dehydrogenase & - & - & - \\
\hline & FTT1564 & - & Polyphosphate kinase & 9 & 6 & 1 \\
\hline \multirow[t]{6}{*}{4} & FTT0018 & - & Secretion protein & 10 & - & - \\
\hline & FTT0296 & Pcp & Pyrrolidone-carboxylate peptidase & - & - & - \\
\hline & FTT0482C & - & Lipoprotein & 32 & 14 & 11 \\
\hline & FTT1029 & DacD & D-Alanyl-D-alanine carboxypeptidase (penicillin binding protein) family protein & 5 & 2 & 2 \\
\hline & FTT1157c & - & Type IV pili lipoprotein & - & - & - \\
\hline & FTT1242 & - & Hypothetical protein & - & - & - \\
\hline
\end{tabular}


Table 2 List of proteins selected for experimental yeast two-hybrid evaluation (Continued)

\begin{tabular}{|c|c|c|c|c|c|c|}
\hline & FTT1591 & VacJ & Lipoprotein & - & - & - \\
\hline & FTT1746 & - & Peptidase & - & - & - \\
\hline \multirow[t]{4}{*}{5} & FTT1597 & - & Putative uncharacterized protein & 16 & 6 & 6 \\
\hline & FTT0522 & - & Hypothetical protein, methyltransferase domain & - & - & - \\
\hline & FTT0604 & - & Hypothetical protein, methyltransferase domain & - & - & - \\
\hline & FTT0677c & - & Putative uncharacterized protein & 2 & - & - \\
\hline
\end{tabular}

Group 1, multiple virulence association evidences and evidence about attenuation in an animal model; group 2, multiple virulence association evidences; group 3, transposon mutant screening evidence only; group 4, predicted secretion evidence only; group 5, orthology evidence only

of 2.62 CFU (Fig. 2 and Table 3) agreed with previously estimated doses for the wild-type strain [9]. Table 3 also shows that the estimated $\mathrm{LD}_{50}$ values for $\triangle \mathrm{FTT} 0482 \mathrm{c}$, $\Delta$ FTT1538c, and $\Delta$ FTT1597 were above the wild-type dose. Further analysis showed that the $\mathrm{LD}_{50}$ values for these three mutant strains were statistically larger ( $p$-value $\leq 0.03$ ) than the $\mathrm{LD}_{50}$ values of either the wild-type strain or the other two mutant strains (Additional file 6: Table S4).

The FTT0902 mutant showed a minute absolute increase in virulence that may be testing the limit of the experimental setup in terms of measureable CFUs. Although FTT0902 does not code for a known protein, it appears to have lipoprotein characteristics based on inspection of the amino terminus, potentially affecting the outer surface of $F$. tularensis. Such minor morphological changes may affect clumping of cells during the preparation of challenge doses and lead to an undercounting of the true dose. Conversely, the possibility that this mutant was really more virulent in the murine model of tularemia could not be ruled out based on our experiments, and, hence, we did not consider $\Delta$ FTT0902 for further evaluation.

Given these results, we compared the survival curves of mice exposed to the $\Delta$ FTT0482c, $\Delta$ FTT1538c, and $\Delta$ FTT1597 mutant strains for intranasal doses of $\geq 78 \mathrm{CFU}$ $\left(\geq 30 \mathrm{LD}_{50}\right)$ to mice exposed to a wild-type strain dose of $320 \mathrm{CFU}\left(122 \mathrm{LD}_{50}\right)$. Figure 3 shows that on the fifth day, all wild-type mice exposed to the high-dose
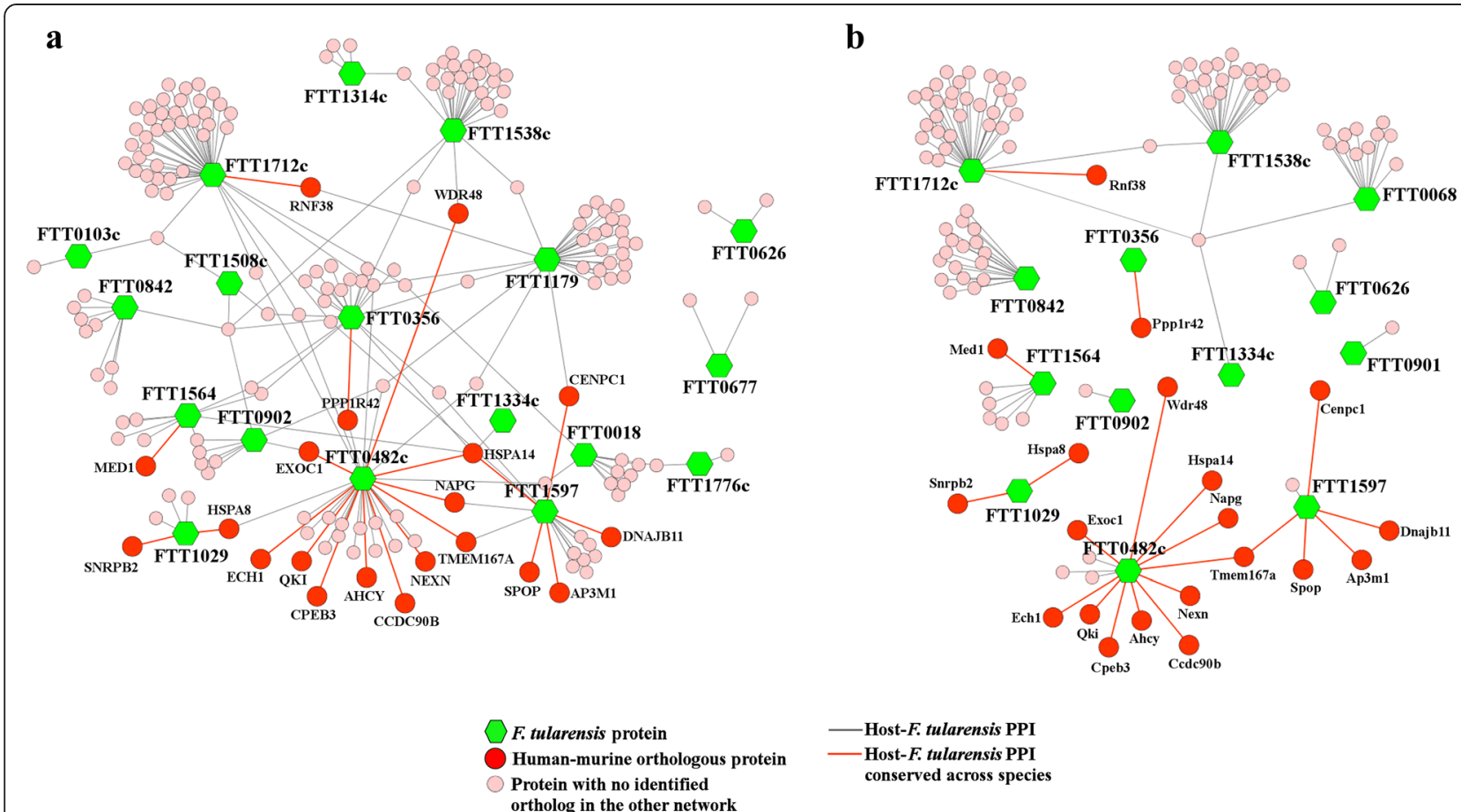

Fig. 1 Yeast two-hybrid (Y2H) host-pathogen protein-protein interactions (PPIs). Using Y2H screens against whole human and murine proteome libraries, we identified 222 unique PPIs between 18 Francisella tularensis proteins and 183 human proteins (a) and 118 unique PPIs between 13 F. tularensis proteins and 113 murine proteins $(\mathbf{b})$. Green nodes represent $F$. tularensis proteins, whereas pink and red nodes represent host proteins. Eleven F. tularensis proteins interacted with both hosts, and six of them participated in 21 conserved interactions (red edges), i.e., six F. tularensis proteins that interacted with both human proteins (red nodes in $\mathbf{a}$ ) and their murine orthologs (red nodes in $\mathbf{b}$ ) 


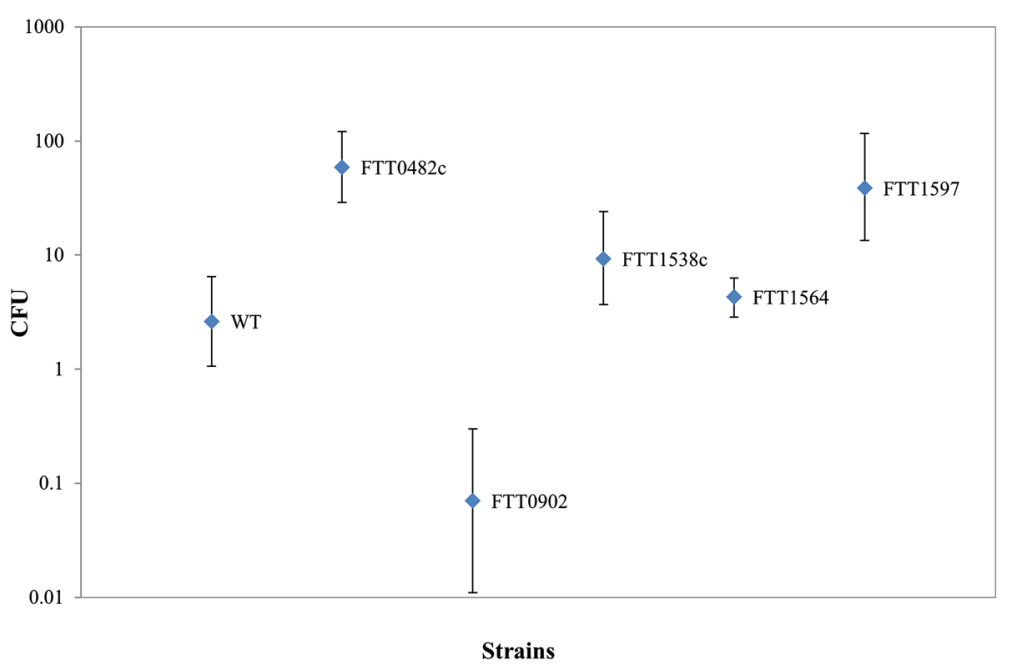

Fig. 2 Fifty percent lethal dose $\left(L D_{50}\right)$ estimation. Using the dose-response experiments, we estimated $L D_{50}$ values and $95 \%$ confidence intervals for the wild-type strain and each of the mutant strains. The estimated wild-type LD 50 of 2.62 CFU, where CFU denote colony-forming units, agreed with previously estimated values for the wild-type $F$. tularensis subsp. tularensis Schu S4 strain. The estimated $\mathrm{LD}_{50}$ values for $\Delta \mathrm{FTT} 0482 \mathrm{C}$ and $\Delta \mathrm{FTT} 1597$ were higher than the wild-type value and outside its $95 \%$ confidence intervals, whereas the $\mathrm{LD}_{50}$ value estimated for $\Delta \mathrm{FTT} 1538 \mathrm{C}$ was above the wild-type value, although with overlapping confidence intervals. These results support the notion that $\Delta F T T 0482 C, \Delta F T T 1538 C$, and $\Delta$ FTT1597 attenuate $F$. tularensis virulence in this animal model

died. Conversely, mice exposed to mutant strains tended to have a slower time to death (nine of ten mice exposed to $\Delta$ FTT1538c died by day 9) as well as survived in larger numbers (five mice exposed to $\triangle$ FTT1597 and four mice exposed to $\triangle$ FTT0482c survived 21 days post-exposure). A survival-curve analysis indicated that the difference in survival times between mutant strains and the wild-type strain was statistically significant $\left(p\right.$-value $\left.\leq 10^{-3}\right)$. Although the values in terms of CFUs are modest for the three mutants, and may not be of biological significance for FTT1538c, all results showed a consistent increase in CFUs. Thus, these results demonstrate that these three mutants showed attenuated virulence when used to challenge $B A L B / c$ mice via the intranasal route of infection and, therefore, implicated three F. tularensis proteins not previously considered factors for in vivo virulence.

\section{Pairwise $\mathrm{Y} 2 \mathrm{H}$ experiments for select human-F. tularensis virulence factors PPIs}

To further assess confidence in the identified hostpathogen interactions from our high-throughput $\mathrm{Y} 2 \mathrm{H}$ screen, we retested a small number of host- $F$. tularensis protein interactions using pairwise $\mathrm{Y} 2 \mathrm{H}$ assays. We focused only on interactions including the identified novel virulence factors (FTT0482c, FTT1538c, and FTT1597) and their interacting human protein partners. To select the human protein interaction partners from the larger data set, we examined and ranked each protein based on involvement in biological processes or pathways associated with $F$. tularensis pathogenicity, conservation among species, and ability to interact with more than one of the three virulence factors. Using this procedure, we selected 12 human-F. tularensis interactions for retesting and confirmed four interactions in this assay: FTT0482c with WD repeat-containing protein 48 (WDR48), FTT1538c with

Table $3 L_{50}$ estimation

\begin{tabular}{|c|c|c|c|c|c|c|}
\hline \multirow[t]{2}{*}{ Strain } & \multicolumn{3}{|c|}{$\log _{10}(\mathrm{CFU}) 95 \%$ confidence intervals } & \multicolumn{3}{|c|}{ CFU $95 \%$ confidence intervals } \\
\hline & Median & Lower & Upper & Median & Lower & Upper \\
\hline Wild type & 0.42 & 0.02 & 0.81 & 2.62 & 1.06 & 6.43 \\
\hline$\Delta \mathrm{FTT} 0482 \mathrm{C}$ & $1.77^{\mathrm{a}}$ & 1.46 & 2.08 & $59.14^{\mathrm{a}}$ & 28.82 & 121.04 \\
\hline$\Delta \mathrm{FTT} 0902$ & -1.14 & -2.01 & -0.52 & 0.07 & 0.01 & 0.30 \\
\hline$\Delta \mathrm{FTT} 1538 \mathrm{c}$ & $0.97^{\mathrm{a}}$ & 0.57 & 1.38 & $9.26^{\mathrm{a}}$ & 3.68 & 24.16 \\
\hline$\Delta \mathrm{FTT} 1564$ & 0.63 & 0.46 & 0.80 & 4.29 & 2.86 & 6.26 \\
\hline$\Delta \mathrm{FTT} 1597$ & $1.59^{\mathrm{a}}$ & 1.13 & 2.07 & $38.66^{a}$ & 13.36 & 116.48 \\
\hline
\end{tabular}

Estimated $50 \%$ lethal dose $\left(\mathrm{LD}_{50}\right)$ values for the $F$. tularensis subsp. tularensis Schu S4 wild-type strain and the following five mutant strains: $\Delta \mathrm{FTT} 0482 \mathrm{c}, \Delta \mathrm{FTT} 0902$, $\Delta \mathrm{FTT1538c,} \Delta \mathrm{FTT1564}$, and $\Delta \mathrm{FTT} 1597$. CFU, colony-forming units. ${ }^{a} \mathrm{LD}_{50}$ values above the wild-type $\mathrm{LD}_{50}$ value 


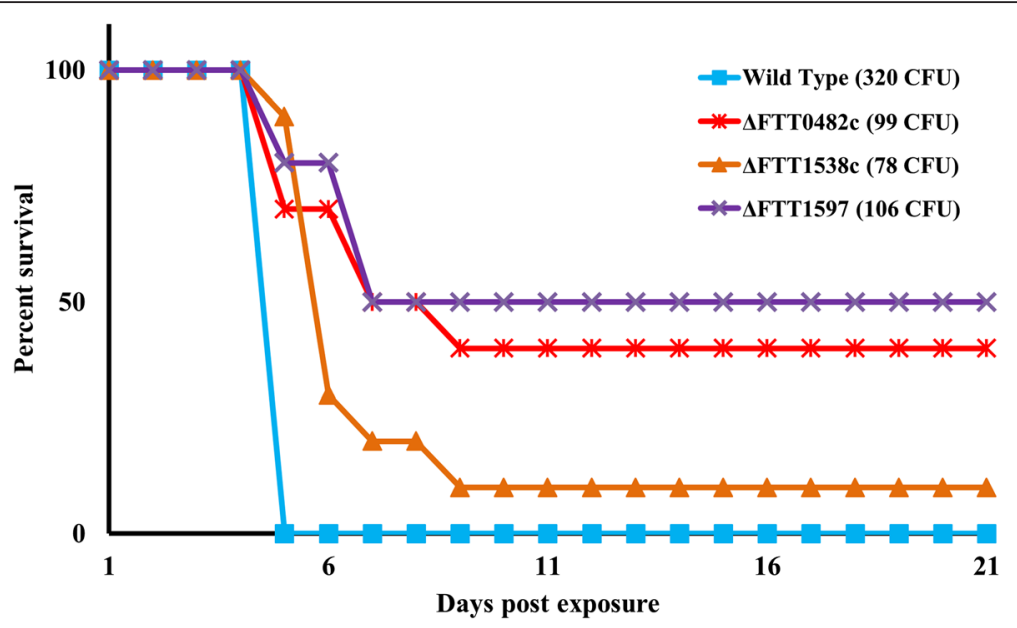

Fig. 3 Mouse intranasal challenge model results. We compared the survival rates of $30(3 \times 10)$ mice exposed to the $\Delta F T T 0482 C, \Delta F T T 1538 c$, and $\Delta$ FTT1597 mutants for intranasal doses of $\geq 78$ CFUs, with 10 mice exposed to wild-type strain doses of 320 CFU. Animals were monitored for 21 days. All mice exposed to the high-dose of the wild-type strain died by the end of the fifth day (blue line), whereas mice exposed to mutant strains had a slower time to death and survived in larger numbers 21 days post-exposure (purple, red, and orange lines). There was a statistically significant difference in the survival rate of mice exposed to mutant strains and mice exposed to the wild-type strain $\left(p\right.$-value $\left.\leq 10^{-3}\right)$. These results support the notion that each of the three mutants attenuates virulence when infected in mice via the intranasal route of infection

78-kDa glucose-regulated protein (HSPA5), FTT1538c with WDR48, and FTT1597 with AP-3 complex subunit mu-1 (AP3M1). The success rate of $33 \%$ was less than the expected $50-80 \%$ rate, although not necessarily out of line with earlier reports of retesting $\mathrm{Y} 2 \mathrm{H}$ interactions using similar assay conditions [55-57]. One reason for the lower reproducibility rate was that the isoforms of the genes used for the retests might not be identical to what we identified in the high-throughput cDNA library screening, as the sequencing reads for the preys provide information for only part of the cDNA. In the retesting, we used the additionally constructed human clones to identify three new interactions that were not detected in our original high-throughput screening: FTT1538c/AP3M1, FTT1597/WDR48, and FTT1597/Quaking (QKI). All retest results are shown in supplemental information (Additional file 7: Table S5).

The pairwise retesting of the $\mathrm{Y} 2 \mathrm{H}$ interactions does not constitute a methodology-independent verification of the initial screening results. We attempted two independent approaches to verify select interaction from the $\mathrm{Y} 2 \mathrm{H}$ assays, i.e., 1) one bait-one prey interaction verification by recombinant expression of bait and prey genes followed by co-immunoprecipitation and LUminescence-based Mammalian IntERactome (LUMIER) assays and 2) affinity purification/mass spectrometry (AP/MS) assays. We restricted these studies to three F. tularensis targets - FTT1597, FTT0482c, and FTT1564 - that interacted with four human proteins. Although we successfully cloned all three selected $F$. tularensis genes and three of the four selected human genes into the LUMIER expression vectors and transfected them into the human HEK293T cell line, we were essentially unable to express the bacterial proteins using multiple expression vectors. It may not be biologically feasible to express the selected bacterial genes in mammalian cell lines, as complex folding and posttranslational processing may not be reproducible in the in vitro environment.

\section{Discussion}

\section{Using $\mathrm{Y} 2 \mathrm{H}$ methods to detect Francisella-human protein interactions}

Protein-interaction detection based on random cDNA libraries and $\mathrm{Y} 2 \mathrm{H}$ technology is associated with wellknown experimental and biological biases leading to mixed confidence and confusion about the reliability of the observed interactions. Prime is the often-noted nonrepeatability of observed $\mathrm{Y} 2 \mathrm{H}$ interactions, primarily stemming from insufficient retesting [58]. It is not practical to repeat screens exhaustively to recover all possible $\mathrm{Y} 2 \mathrm{H}$ interaction high-throughput experiments. Although more testing increases confidence of multiple observed interactions, the absence of an interaction may not indicate that the interaction cannot occur, just that the experimental or biological conditions were not amenable to sample that interaction. A further uncertainty on the biological level is that presence of an $\mathrm{Y} 2 \mathrm{H}$ interaction does not necessarily indicate that the interaction actually occurs, just that it can occur.

Comparison of high-throughput data sets. Our study is not the first study to screen for interactions between human and F. tularensis proteins. However, it is the first study to systematically use targeted screens to identify 
potential F. tularensis virulence factors and PPIs associated with secretion system proteins. In contrast, Dyer et al. [59] used a random genome library of $F$. tularensis as baits (DNA-binding domain) and human cDNA libraries as preys (activation domain) to identify host-pathogen interactions using $\mathrm{Y} 2 \mathrm{H}$ technology. This study reported 1,383 host-pathogen interactions between 999 human and $415 \mathrm{~F}$. tularensis proteins. We found that 24 proteins from our list of $49 \mathrm{~F}$. tularensis putative and known virulence factors were present in the Dyer et al. data set, with nine proteins associated with 180 interactions in our data set. Although these nine proteins participated in 43 PPIs in the Dyer et al. study, there was no overlap among these interactions and our data set. This result is again a consequence of the variations in the libraries, $\mathrm{Y} 2 \mathrm{H}$ vectors, and strains used in terms of different bait/ prey clone properties and laboratory procedures. For example, Dyer et al. screened random fragments of $F$. tularensis genomic DNA, whereas we used full-length ORF clones. Similar low overlaps have been noted among Helicobacter pylori proteins [56] and for Yersinia pestis host-pathogen PPIs obtained by random library and systematic $\mathrm{Y} 2 \mathrm{H}$ screening [60].

\section{Analysis of virulence factor interactions and infectivity mechanisms}

We have chosen to organize the discussion of virulence factor interactions with host proteins into three sections (I-III) based on the level of interaction confidence. Thus, the first analysis was based on highest confidence data, consisting of the successfully retested $\mathrm{Y} 2 \mathrm{H}$ interactions, for the three novel in vivo validated virulence factors identified here. Second, we used all $\mathrm{Y} 2 \mathrm{H}$ data for the two previously known virulence factors included in our data set to assess their possible contribution to Francisella pathogenicity. Finally, we present an analysis of potential Francisella infectivity mechanisms that factors in all detected $\mathrm{Y} 2 \mathrm{H}$ interactions in identifying targeted human proteins and protein interaction networks.

\section{I - Novel virulence factors and their PPI-inferred virulence phenotypes}

Little prior knowledge exists on the function or role of virulence factor proteins FTT0482c, FTT1538c, and FTT1597; only FTT0482c has been assigned functionality as a hypothetical lipoprotein. Here, we positively identified them as virulence factors that do not influence normal bacterial growth yet affect lethality in an intranasal murine challenge model. Furthermore, we can hypothesize on their possible roles using the measured $\mathrm{Y} 2 \mathrm{H}$ interaction profiles with human proteins in promoting Francisella intracellular lifestyle and pathogenicity.
The interactions of FTT1538c with AP3M1, WDR48, and HSPA5 strongly link this protein to biological processes involved in human intracellular transport. AP3M1 is a component of an adaptor complex involved in the Golgi to endosome/lysosome intracellular protein trafficking, in particular from the early endosome to the late endosome compartment [61]. Binding to and interfering with this adaptor complex could alter endosome maturation or endosome-lysosome fusion. Similarly targeting lysosomal degradation processes, FTT1538c binding or interacting with WDR48, known to regulate deubiquitinating complexes [62], could provide a mechanism to interfere with ubiquitin tagging of pathogen protein and prevent their targeted destruction. HSPA 5 belongs to the family of $70-\mathrm{kDa}$ heat shock proteins generally found in the endoplasmic reticulum; however, it is associated with multiple cellular locations and functions, including the unfolded protein response, protein transport, and interactions with apoptotic executors [63].

Consistent with these interactions/targets, host targets identified in the high-throughput $\mathrm{Y} 2 \mathrm{H}$ include SNARE protein STX8, a protein known to be required for late endosome trafficking [63], and PI4K2B, involved in overall phosphatidylinositol 4-kinase activity and protein trafficking [64]. Furthermore, GO enrichment analysis of all host proteins that interact with FTT1538c also indicated enrichment of membrane-bound vesicular proteins $\left(p_{a d j}=0.05\right)$. Thus, the interaction data are compatible with FTT1538c contributing to intracellular survival by interfering with vesicular trafficking, possibly during endosome and lysosome fusion during phagocytosis, and ultimately contributing to phagosomal escape.

Although lacking functional annotation, FTT1597 contains tetratricopeptide repeat motifs recently shown to be present in other proteins implicated in F. tularensis virulence [65]. The similarity in interactions with AP3M1 and WDR48 highlight a possible functional similarity to FTT1538c. Further linking the functionalities, in the high-throughput Y2H data set, FTT1597 interacted with DNAJB11, a co-chaperone of HSPA5. On the other hand, the observed interaction with QK1, an RNA-binding protein that regulates mRNA activities, is not known to be linked to any of these functionalities. Lipoprotein FTT0482c interacted with WDR48, which thus was a common host interaction among all the identified virulence factors. Five other interacting host proteins in high-throughput screening share this involvement in targeting ubiquitination related proteins/processes (ANAPC10, CACYBP, RANBP2, UBE2V2, and $\mathrm{XPA})$. In these experiments, FTT0482c also interacted with the renin receptor ATP6AP2, which activates the ERK inflammatory pathway and whose inhibition is associated with decreased phagocytosis [66]. 


\section{II - Phenotypic associations of known virulence factors}

Turning to the interaction analysis of known virulence factors based on the high-throughput $\mathrm{Y} 2 \mathrm{H}$ screens, we examined identified PPIs for known virulence factors and those highly implicated in virulence for binding to host protein targets. A lack of host protein interactions does not necessarily imply a lack of a biological role in virulence, as virulence factor proteins may be involved in functions other than protein binding as detectable by the $\mathrm{Y} 2 \mathrm{H}$ screens.

Our screens identified a total of 76 and 12 interactions for FTT1712c (IglC2) and FTT0068 (SodB), respectively (Table 2). IglC2 interacted with proteins from both hosts, whereas SodB interacted only with murine proteins. IglC2 is a Francisella pathogenicity island protein (and a member of T6SS), which has already been identified as a protein required for intracellular growth, phagosomal escape, regulation of host signaling, and apoptosis [7, 67-69]. We identified 43 interactions between $\mathrm{IglC} 2$ and human proteins and 33 interactions between $\mathrm{IglC} 2$ and murine proteins, the largest number of interactions detected for any $F$. tularensis protein in our screens. We found that IglC2 interacted with a set of proteins that are linked to the inflammatory response and activation of cAMP signaling pathway stimulation via mitogen-activated protein kinases, i.e., phosphatidylinositol 3,4,5-trisphosphate 3-phosphatase (PTEN), fibroblast growth factor receptor substrate 2 (FRS2), and transcription factor AP-1 (JUN). As these proteins are intimately linked to host cell defense and immune responses [10, 70], they provide important targets for immune suppression and the successful establishment of an infection.

For SodB that has been identified as a factor in F. tularensis oxidative stress protection [71], we found multiple potential interacting partners, including interactions with the murine thioredoxin-interacting protein Txnip, a protein mediating oxidative stress via thioredoxin activity [72].

Furthermore, we identified interactions for five additional proteins from the first group of proteins, i.e., those highly implicated in virulence (Table 2). For two of those, heat shock protein FTT0356 (HtpG) [11] and GTP-binding translational elongation factor FTT1179 (BipA) [43], we found interactions with human proteins but only one murine interaction, even though murine orthologs exists. For the remaining three proteins (LpnA, RelA, and Lon) we only found a scatter of interactions that did not point toward any specific protein interaction pattern or biological process.

\section{III - Characterization of $F$. tularensis infectivity based on its host targets}

It was not possible to perform a statistically meaningful evaluation of the roles of each individual $F$. tularensis protein due to the overall small number of identified host-pathogen protein interactions. Instead, given that the selected set of 49 bacterial proteins was strongly biased toward secreted/virulence associated protein, we used the aggregated host-pathogen interaction data set to investigate host molecular mechanisms targeted by the selected subset of F. tularensis proteins. The aggregated data set contains all experimentally detected human-F. tularensis PPIs and computationally derived human (murine orthologs)-F. tularensis PPIs and consists of 298 unique interactions between 18 F. tularensis and 249 human proteins.

First, we performed enrichment analyses based on GO annotation as outlined in the METHODS. Table 4 shows that $F$. tularensis proteins target a statistically significant number of host proteins located in or around the endoplasmic reticulum and Golgi compartments. Known pathogen targets in these compartments are linked to activation of protein degradation processes [73].

Second, we used the host PPI network to investigate the influence of the pathogen on host proteins and interactions between host proteins [20]. We mapped proteins from the merged human- $F$. tularensis PPI data set onto a human PPI network consisting of 76,043 physical PPIs among 11,688 proteins [41]. Of the 249 human proteins interacting with $F$. tularensis proteins, $194(\sim 78 \%)$ were found in the human network. Furthermore, we found that 52 of these human proteins were a part of the LCC, i.e., the largest subnetwork in which a path connects every two proteins to each other. The size of this LCC was significantly larger than what could be expected by random chance in the human PPI network, i.e., $N_{\text {ran }}=9.1, \sigma_{\text {ran }}=6.4$. Within this LCC, we identified 139 sets of human PPIs in which, in each set, all human proteins interacted with at least one of the $18 \mathrm{~F}$. tularensis proteins and had the same GO biological process annotations; we denoted these sets as interaction modules [20]. Table 5 shows 12 interaction modules that correspond to the most specific (lowest level) GO annotations of all identified interaction modules. These interaction modules are associated with biological processes related to cellular regulation, signaling, cellular response, and protein modification.

It has been shown that a number of bacterial pathogens interfere with host signaling pathways to obstruct host defense systems and promote pathogen colonization [74-76]. Indeed, the largest number of proteins in the LCC was involved in signaling, and we identified two statistically significant signaling interaction modules, containing eight and five host proteins (Fig. 4, shaded areas). The larger interaction module contained proteins related to defense responses, whereas the smaller interaction module mostly contained proteins related to intracellular signaling. Our analysis also identified 18 
Table 4 Enrichment of $\mathrm{GO}$ terms for human proteins interacting with $F$. tularensis

\begin{tabular}{|c|c|c|c|c|c|c|}
\hline \multirow{2}{*}{ Cellular localization } & \multirow{2}{*}{$\frac{\text { GO term ID }}{\text { GO:0005793 }}$} & \multirow{2}{*}{$\begin{array}{l}\text { GO term description } \\
\text { Endoplasmic reticulum-Golgi intermediate compartment }\end{array}$} & \multirow{2}{*}{ Number of proteins } & \multicolumn{2}{|c|}{$p$-value } & \multirow{2}{*}{$\frac{\mathrm{FDR}}{0.04}$} \\
\hline & & & & 2.7 & $10^{-3}$ & \\
\hline & GO:0005788 & Endoplasmic reticulum lumen & 6 & 1.7 & $10^{-3}$ & 0.03 \\
\hline & GO:0005801 & Cis-Golgi network & 3 & 3.6 & $10^{-3}$ & 0.05 \\
\hline & GO:0005720 & Nuclear heterochromatin & 3 & 3.6 & $10^{-3}$ & 0.05 \\
\hline & GO:0042470 & Melanosome & 7 & 9.5 & $10^{-5}$ & 0.00 \\
\hline & GO:0042588 & Zymogen granule & 3 & 3.3 & $10^{-3}$ & 0.01 \\
\hline & GO:0035097 & Histone methyltransferase complex & 4 & 3.8 & $10^{-3}$ & 0.05 \\
\hline & GO:0031519 & PcG protein complex & 3 & 5.1 & $10^{-3}$ & 0.06 \\
\hline \multirow[t]{2}{*}{ Molecular function } & GO:0051082 & Unfolded protein binding & 6 & 1.9 & $10^{-3}$ & 0.18 \\
\hline & GO:0019904 & Protein domain specific binding & 14 & 3.6 & $10^{-3}$ & 0.18 \\
\hline
\end{tabular}

FDR, false discovery rate calculated using Benjamini and Hochberg multiple test correction [40]; GO, Gene Ontology

stress response proteins in the LCC, 16 of which were also annotated as signaling proteins. One of the remaining two proteins was aldo-keto reductase family 1 protein (AKR1B1), a protein involved in the stress response [77], which links the two interaction modules. The other stress response protein, chromobox protein homolog 5 (CBX5), links the larger signaling interaction module and signal transducing adapter molecule 2 (STAM2), which is associated with the immune system response. Further assessment of proteins from the LCC revealed additional proteins associated with signaling (blue octagons) [78]. Overall, the identified signaling stress response component consists of $50 \%$ of proteins from the LCC (26 proteins). These results suggest that proteins involved in cell-to-cell signaling and innate immune defense responses are potential targets for Francisella; furthermore, it also points toward the interactions among these host proteins as equally important targets.

\section{Conclusions}

Direct interactions with specific host proteins and the ability to influence host PPIs are important components for intracellular pathogens to avoid host-cell defense mechanisms and successfully establish an infection. Although direct host-pathogen protein-protein binding is only one aspect of virulence, it is a critical component in directly manipulating and interfering with cellular processes in the host cell. Here, we exploited this using an integrated bioinformatics/proteomics method

Table 5 Enrichment of GO biological processes in host subnetworks

\begin{tabular}{|c|c|c|c|c|c|c|c|c|c|c|}
\hline \multirow[t]{2}{*}{ Category } & \multicolumn{2}{|l|}{ Term } & \multicolumn{2}{|c|}{ Size } & \multicolumn{6}{|c|}{$p$-value } \\
\hline & $\mathrm{ID}$ & Description & $N_{C}$ & $N_{\mathrm{M}}$ & $\mathrm{P}_{\mathrm{GO}}$ & & $\mathrm{P}_{\mathrm{Rp}}$ & & $\mathrm{P}_{\mathrm{Rn}}$ & \\
\hline \multirow[t]{5}{*}{ Cellular regulation } & GO:0045944 & Positive regulation of transcription from RNA polymerase II promoter & 7 & 4 & 6.4 & $10^{-3}$ & 1.0 & $10^{-4}$ & 1.5 & $10^{-4}$ \\
\hline & GO:0045892 & Negative regulation of transcription, DNA-dependent & 11 & 6 & 0.0 & & 0.0 & & 1.3 & $10^{-4}$ \\
\hline & GO:0051090 & Regulation of sequence-specific DNA binding transcription factor activity & 6 & 4 & 1.1 & $10^{-3}$ & 0.0 & & 1.1 & $10^{-4}$ \\
\hline & GO:0051345 & Positive regulation of hydrolase activity & 8 & 5 & 4.0 & $10^{-4}$ & 0.0 & & 7.7 & $10^{-5}$ \\
\hline & GO:0051726 & Regulation of cell cycle & 9 & 4 & 6.0 & $10^{-4}$ & 7.0 & $10^{-4}$ & 1.1 & $10^{-3}$ \\
\hline \multirow[t]{2}{*}{ Cellular signaling } & GO:0023052 & Signaling & 22 & 8 & 9.2 & $10^{-3}$ & 0.0 & & 1.1 & $10^{-3}$ \\
\hline & GO:0007166 & Cell surface receptor signaling pathway & 15 & 5 & 3.4 & $10^{-3}$ & 6.0 & $10^{-4}$ & 1.8 & $10^{-3}$ \\
\hline \multirow[t]{3}{*}{ Cellular response } & GO:0070887 & Cellular response to chemical stimulus & 17 & 7 & 0.0 & & 0.0 & & 4.0 & $10^{-4}$ \\
\hline & GO:0006950 & Response to stress & 18 & 10 & 4.0 & $10^{-3}$ & 0.0 & & 4.5 & $10^{-4}$ \\
\hline & GO:0010033 & Response to organic substance & 17 & 8 & 0.0 & & 0.0 & & 4.6 & $10^{-4}$ \\
\hline \multirow[t]{2}{*}{ Protein modification } & GO:0016567 & Protein ubiquitination & 9 & 4 & 0.0 & & 1.0 & $10^{-4}$ & 2.9 & $10^{-5}$ \\
\hline & GO:0016310 & Phosphorylation & 10 & 5 & 9.7 & $10^{-3}$ & 2.0 & $10^{-4}$ & 1.1 & $10^{-4}$ \\
\hline
\end{tabular}

$N_{C}$, number of proteins in the largest connected component annotated with a given term; $N_{M}$, number of proteins in the largest interaction module for a given term; $\mathrm{p}_{\mathrm{GO}}$, probability of the same number of proteins as $N_{\mathrm{C}}$ being annotated with a given $\mathrm{GO}$ term solely through a random selection; $\mathrm{p}_{\mathrm{Rn}}$, probability that a given number of proteins as $N_{M}$ are annotated with a given $\mathrm{GO}$ term solely through random selection in a random network that has the same degree distribution as the human network; $p_{R p}$, probability that a given number of proteins as $N_{M}$ are annotated with a given $G O$ term solely through random selection. This table contains only the largest statistically significant interaction module for each term; the complete list is available as supplementary information (Additional file 8: Table S6). 


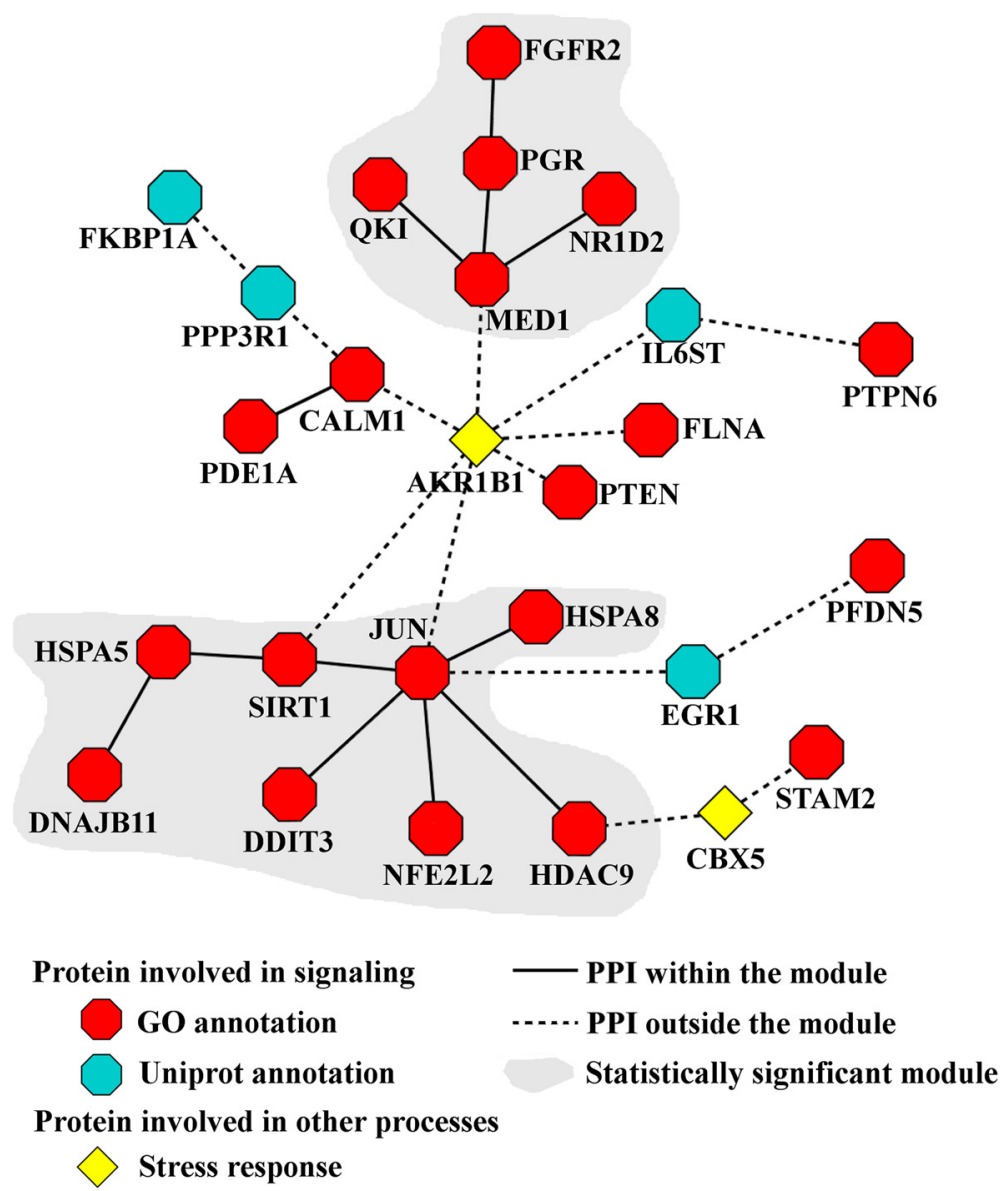

Fig. 4 Host signaling and the stress response as a F. tularensis virulence factor target. F. tularensis proteins target a large number of host proteins involved in signaling (red and blue octagons) and the stress response (yellow diamond). Using the connectivity and Gene Ontology (GO) annotation, we identified two statistically significant signaling interaction modules, one containing eight host proteins and one containing five host proteins (shaded area). The larger one contains proteins related to the immune system response, whereas the smaller one contains proteins related to intracellular signaling. Further annotation assessment of proteins from the largest connected component (LCC) revealed additional proteins associated with signaling (blue octagons) and existing interaction modules. These results suggest that $F$. tularensis targets host proteins involved in signaling to interfere with cell-to-cell signaling and the immune response but also points toward PPIs among these host proteins as equally important targets

to identify both virulence factors and their complement of interacting host proteins.

The implemented approach of selecting a focused set of putative virulence factors based on multiple evidence, down-selection through in vitro highthroughput $\mathrm{Y} 2 \mathrm{H}$ screening, and final assessment using mutants in an animal infection model was highly effective. Of the final five mutants selected for animal testing, three showed statistically significant virulence attenuation that was not directly attributable to gross bacterial growth defects of the tested mutants. Furthermore, the up-front $\mathrm{Y} 2 \mathrm{H}$ screening and subsequent retesting of select host-pathogen protein interactions provided indications as to possible virulence mechanisms associated with the direct binding of the virulence factors to host proteins. This led to an initial hypothesis as to which host processes were targeted by the three virulence factors FTT0482c, FTT1538c, and FTT1597, in particular identifying intracellular protein trafficking between the early endosome to the late endosome as an important component in virulence attenuation. We also used the $\mathrm{Y} 2 \mathrm{H}$ data to investigate host protein binding of two known virulence factors, showing that direct protein binding was a component in the modulation of the inflammatory response via activation of mitogen-activated protein kinases and in the oxidative stress response.

\section{Availability of supporting data}

The data sets supporting the results of this article are included within the article and its additional files. 


\section{Additional files}

Additional file 1: Table S1. Francisella strains used to identify proteins overrepresented in the pathogenic strains. (DOCX $27 \mathrm{~kb}$ )

Additional file 2: PPI data. F. tularensis protein interactions with 1) human proteins, 2) murine proteins, and 3) the combined set of human proteins augmented with murine orthologs. (XLSX 28 kb)

Additional file 3: Table S2. Exponential-phase growth rates. (DOCX $20 \mathrm{~kb}$ ) Additional file 4: Table S3. Mouse intranasal infection results. (DOCX $28 \mathrm{~kb}$ )

Additional file 5: Figure S1. Evaluation of the effect of five mutants on Francisella tularensis virulence using mouse intranasal model experiments. (DOCX 372 kb)

Additional file 6: Table S4. Likelihood that one strain is more or less potent than any other strain. (DOCX $18 \mathrm{~kb}$ )

Additional file 7: Table S5. Human- $F$. tularensis protein-protein interactions retested in pairwise $\mathrm{Y} 2 \mathrm{H}$ experiments. (DOCX $20 \mathrm{~kb}$ )

Additional file 8: Table S6. Enrichment of $\mathrm{GO}$ biological processes in host subnetworks. (DOCX $63 \mathrm{~kb}$ )

\section{Abbreviations}

3-AT: 3-amino-1,2,4-triazole; AKR1B1: Aldo-keto reductase family 1 protein AP3M1: AP-3 complex subunit mu-1; CBX5: Chromobox protein homolog 5 CFU: Colony-forming unit; ETA: Enhanced tularemia agar; ETB: Enhanced tularemia broth; FRS2: Fibroblast growth factor receptor substrate 2; GO: Gene Ontology; HSPA5: 78-kDa glucose-regulated protein; IBC: Institutional Biosafety Committee; JUN: Transcription factor AP-1; LCC: Largest connected component; LD 50 : 50 \% lethal dose; NCBI: National Center for Biotechnology Information; $\mathrm{OD}_{600}$ : Optical density measurements at $600 \mathrm{~nm}$; ORF: Open reading frame; PPI: Protein-protein interaction; PTEN: Phosphatidylinositol 3,4,5-trisphosphate 3-phosphatase; QKI: Quaking; STAM2: Signal transducing adapter molecule 2; T2SS: Type II secretion system; T6SS: Type VI secretion system; USAMRIID: United States Army Medical Research Institute for Infectious Diseases; WDR48: WD repeat-containing protein 48; Y2H: Yeast two-hybrid; YEPDA: Yeast extract, peptone, dextrose, and adenine.

\section{Competing interests}

The authors declare that they have no competing interests.

\section{Authors' contributions}

$N Z, R P, S V R, J R$, and AW designed the research; VM, NZ, RP, SVR, KK, and TAH performed research; VM, NZ, CY, SVR, RP, TAH, and AW analyzed data; and $A W, V M, R P, S V R$, and TAH. wrote the paper; all authors edited the paper and JR edited the final version of the paper. All authors read and approved the final manuscript.

\section{Acknowledgements}

This work was supported by U.S. Defense Threat Reduction Agency Award CBS.MEDBIO.02.10.BH.021 and by the U.S. Army Medical Research and Materiel Command (Ft. Detrick, MD) as part of the U.S. Army's Network Science Initiative. The opinions and assertions contained herein are the private views of the authors and are not to be construed as official or as reflecting the views of the U.S. Army or the U.S. Department of Defense. This paper has been approved for public release with unlimited distribution.

\section{Author details}

${ }^{1}$ Department of Defense Biotechnology High Performance Computing Software Applications Institute, Telemedicine and Advanced Technology Research Center, U.S. Army Medical Research and Materiel Command, Fort Detrick, MD 21702, USA. ${ }^{2}$ J. Craig Venter Institute, Rockville, MD 20850, USA. ${ }^{3}$ Bacteriology Division, U.S. Army Medical Research Institute of Infectious Diseases, Fort Detrick, MD 21702, USA

Received: 30 July 2015 Accepted: 21 December 2015 Published online: 29 December 2015

\section{References}

1. McLendon MK, Apicella MA, Allen LA. Francisella tularensis: taxonomy, genetics, and immunopathogenesis of a potential agent of biowarfare. Annu Rev Microbiol. 2006;60:167-85.

2. Dennis DT, Inglesby TV, Henderson DA, Bartlett JG, Ascher MS, Eitzen E, et al. Tularemia as a biological weapon: medical and public health management. JAMA, J Am Med Assoc. 2001;285(21):2763-73.

3. Asare R, Kwaik YA. Exploitation of host cell biology and evasion of immunity by Francisella tularensis. Front Microbiol. 2011;1:145.

4. Chong A, Celli J. The Francisella intracellular life cycle: toward molecular mechanisms of intracellular survival and proliferation. Front Microbiol. 2010;1:138.

5. Dieppedale J, Sobral D, Dupuis M, Dubail I, Klimentova J, Stulik J, et al. Identification of a putative chaperone involved in stress resistance and virulence in Francisella tularensis. Infect Immun. 2011;79(4):1428-39.

6. Lindemann SR, Peng K, Long ME, Hunt JR, Apicella MA, Monack DM, et al. Francisella tularensis Schu S4 O-antigen and capsule biosynthesis gene mutants induce early cell death in human macrophages. Infect Immun. 2011;79(2):581-94.

7. Lindgren H, Golovliov I, Baranov V, Ernst RK, Telepnev M, Sjostedt A. Factors affecting the escape of Francisella tularensis from the phagolysosome. J Med Microbiol. 2004;53(Pt 10):953-8.

8. Moreau GB, Mann BJ. Adherence and uptake of Francisella into host cells. Virulence. 2013:4(8):826-32.

9. Pechous RD, McCarthy TR, Mohapatra NP, Soni S, Penoske RM, Salzman NH, et al. A Francisella tularensis Schu S4 purine auxotroph is highly attenuated in mice but offers limited protection against homologous intranasal challenge. PLoS One. 2008;3(6), e2487.

10. Rajaram MV, Butchar JP, Parsa KV, Cremer TJ, Amer A, Schlesinger LS, et al. Akt and SHIP modulate Francisella escape from the phagosome and induction of the Fas-mediated death pathway. PLoS One. 2009;4(11), e7919.

11. Wehrly TD, Chong A, Virtaneva K, Sturdevant DE, Child R, Edwards JA, et al. Intracellular biology and virulence determinants of Francisella tularensis revealed by transcriptional profiling inside macrophages. Cell Microbiol. 2009:11(7):1128-50.

12. Meibom KL, Charbit A. The unraveling panoply of Francisella tularensis virulence attributes. Curr Opin Microbiol. 2010;13(1):11-7.

13. Nano FE, Zhang N, Cowley SC, Klose KE, Cheung KK, Roberts MJ, et al. A Francisella tularensis pathogenicity island required for intramacrophage growth. J Bacteriol. 2004;186(19):6430-6.

14. Barker JR, Chong A, Wehrly TD, Yu JJ, Rodriquez SA, Liu J, et al. The Francisella tularensis pathogenicity island encodes a secretion system that is required for phagosome escape and virulence. Mol Microbiol. 2009;74(6):1459-70.

15. Broms JE, Meyer L, Sun K, Lavander M, Sjostedt A. Unique substrates secreted by the type VI secretion system of Francisella tularensis during intramacrophage infection. PLoS One. 2012;7(11), e50473.

16. Broms JE, Sjostedt A, Lavander M. The role of the Francisella tularensis pathogenicity island in type VI secretion, intracellular survival, and modulation of host cell signaling. Front Microbiol. 2010;1:136.

17. Salomonsson E, Forsberg A, Roos N, Holz C, Maier B, Koomey M, et al. Functional analyses of pilin-like proteins from Francisella tularensis: complementation of type IV pilus phenotypes in Neisseria gonorrhoeae. Microbiol. 2009:155(Pt 8):2546-59.

18. Forsberg A, Guina T. Type II secretion and type IV pili of Francisella. Ann N Y Acad Sci. 2007;1105:187-201.

19. Memisevic V, Zavaljevski N, Pieper R, Rajagopala SV, Kwon K, Townsend $K$, et al. Novel Burkholderia mallei virulence factors linked to specific host-pathogen protein interactions. Mol Cell Proteomics. 2013;12(11):3036-51.

20. Memisevic V, Zavaljevski N, Rajagopala SV, Kwon K, Pieper R, DeShazer D, et al. Mining host-pathogen protein interactions to characterize Burkholderia mallei infectivity mechanisms. PLoS Comput Biol. 2015;11(3), e1004088.

21. Hachani A, Allsopp LP, Oduko Y, Filloux A. The VgrG proteins are "a la carte" delivery systems for bacterial type VI effectors. J Biol Chem. 2014;289(25):17872-84.

22. Gillespie JJ, Wattam AR, Cammer SA, Gabbard JL, Shukla MP, Dalay O, et al. PATRIC: the comprehensive bacterial bioinformatics resource with a focus on human pathogenic species. Infect Immun. 2011:79(11):4286-98.

23. Yu C, Desai V, Cheng L, Reifman J. QuartetS-DB: A large-scale orthology database for prokaryotes and eukaryotes inferred by evolutionary evidence. BMC Bioinformatics. 2012;13:143. 
24. Yu C, Zavaljevski N, Desai V, Reifman J. QuartetS: a fast and accurate algorithm for large-scale orthology detection. Nucleic Acids Res. 2011;39(13), e88.

25. Pruitt KD, Tatusova T, Brown GR, Maglott DR. NCBI Reference Sequences (RefSeq): current status, new features and genome annotation policy. Nucleic Acids Res. 2012;40(Database issue):D130-5.

26. Altschul SF, Madden TL, Schaffer AA, Zhang J, Zhang Z, Miller W, et al. Gapped BLAST and PSI-BLAST: A new generation of protein database search programs. Nucleic Acids Res. 1997;25(17):3389-402

27. Petersen TN, Brunak S, von Heijne G, Nielsen H. SignalP 4.0: discriminating signal peptides from transmembrane regions. Nat Methods. 2011;8(10):785-6.

28. Stellberger T, Hauser R, Baiker A, Pothineni VR, Haas J, Uetz P. Improving the yeast two-hybrid system with permutated fusions proteins: the Varicella Zoster Virus interactome. Proteome Sci. 2010;8:8.

29. Rajagopala SV, Uetz P. Analysis of protein-protein interactions using high-throughput yeast two-hybrid screens. Methods Mol Biol. 2011;781:1-29.

30. Lamesch P, Li N, Milstein S, Fan C, Hao T, Szabo G, et al. hORFeome v3.1: a resource of human open reading frames representing over 10,000 human genes. Genomics. 2007:89(3):307-15.

31. National Institutes of Health. Mammalian Gene Collection (MGC). 2015. http://mgc.nci.nih.gov/. Accessed 30 March 2015.

32. Bewick V, Cheek $L$, Ball J. Statistics review 12: survival analysis, Crit Care. 2004;8(5):389-94.

33. Harrington DP, Fleming TR. A class of rank test procedures for censored survival data. Biometrika. 1982;69(3):553-66.

34. Sayers EW, Barrett T, Benson DA, Bolton E, Bryant SH, Canese K, et al. Database resources of the National Center for Biotechnology Information. Nucleic Acids Res. 2012;40(Database issue):D13-25.

35. Shannon P, Markiel A, Ozier O, Baliga NS, Wang JT, Ramage D, et al Cytoscape: a software environment for integrated models of biomolecular interaction networks. Genome Res. 2003;13(11):2498-504.

36. Csardi G, Nepusz T. The igraph software package for complex network research. Int J. 2006;Complex Systems:1695.

37. Shannon PT, Grimes M, Kutlu B, Bot JJ, Galas DJ. RCytoscape: Tools for exploratory network analysis. BMC Bioinformatics. 2013;14:217.

38. Ashburner M, Ball CA, Blake JA, Botstein D, Butler H, Cherry JM, et al. Gene Ontology: tool for the unification of biology. Nat Genet. 2000;25(1):25-9.

39. Durinck S, Moreau Y, Kasprzyk A, Davis S, De Moor B, Brazma A, et al. BioMart and Bioconductor: A powerful link between biological databases and microarray data analysis. Bioinformatics. 2005;21(16):3439-40.

40. Benjamini $Y$, Hochberg Y. Controlling the false discovery rate: a practical and powerful approach to multiple testing. J R Stat Soc, Series B (Methodol). 1995;57(1):289-300.

41. Yu X, Wallqvist A, Reifman J. Inferring high-confidence human protein-protein interactions. BMC Bioinformatics. 2012;13:79.

42. Marohn ME, Santiago AE, Shirey KA, Lipsky M, Vogel SN, Barry EM. Members of the Francisella tularensis phagosomal transporter subfamily of major facilitator superfamily transporters are critical for pathogenesis. Infect Immun. 2012;80(7):2390-401.

43. Su J, Yang J, Zhao D, Kawula TH, Banas JA, Zhang JR. Genome-wide identification of Francisella tularensis virulence determinants. Infect Immun. 2007;75(6):3089-101.

44. Weiss DS, Brotcke A, Henry T, Margolis JJ, Chan K, Monack DM. In vivo negative selection screen identifies genes required for Francisella virulence. Proc Natl Acad Sci U S A. 2007;104(14):6037-42.

45. Su J, Asare R, Yang J, Nair MK, Mazurkiewicz JE, Abu-Kwaik Y, et al. The capBCA locus is required for intracellular growth of Francisella tularensis LVS. Front Microbiol. 2011;2:83.

46. Child R, Wehrly TD, Rockx-Brouwer D, Dorward DW, Celli J. Acid phosphatases do not contribute to the pathogenesis of type A Francisella tularensis. Infect Immun. 2010;78(1):59-67.

47. Ark NM, Mann BJ. Impact of Francisella tularensis pilin homologs on pilus formation and virulence. Microb Pathog. 2011;51(3):110-20.

48. Konecna K, Hernychova L, Reichelova M, Lenco J, Klimentova J, Stulik J, et al. Comparative proteomic profiling of culture filtrate proteins of less and highly virulent Francisella tularensis strains. Proteomics. 2010;10(24):4501-11.

49. Luo H, Lin Y, Gao F, Zhang CT, Zhang R. DEG 10, an update of the database of essential genes that includes both protein-coding genes and noncoding genomic elements. Nucleic Acids Res. 2014;42(Database issue):D574-80.

50. Gray KA, Daugherty LC, Gordon SM, Seal RL, Wright MW, Bruford EA. Genenames.org: the HGNC resources in 2013. Nucleic Acids Res. 2013; 41 (Database issue):D545-52.
51. Blake JA, Bult CJ, Eppig JT, Kadin JA, Richardson JE. The Mouse Genome Database: integration of and access to knowledge about the laboratory mouse. Nucleic Acids Res. 2014;42(Database issue):D810-7.

52. Chamberlain RE. Evaluation of live Tularemia vaccine prepared in a chemically defined medium. Appl Microbiol. 1965;13:232-5.

53. Mao X, Ma Q, Zhou C, Chen X, Zhang H, Yang J, et al. DOOR 2.0: presenting operons and their functions through dynamic and integrated views. Nucleic Acids Res. 2014;42(Database issue):D654-9.

54. Taboada B, Ciria R, Martinez-Guerrero CE, Merino E. ProOpDB: Prokaryotic Operon DataBase. Nucleic Acids Res. 2012;40(Database issue):D627-31.

55. Yu X, Ivanic J, Memisevic V, Wallqvist A, Reifman J. Categorizing biases in high-confidence high-throughput protein-protein interaction data sets. Mol Cell Proteomics. 2011;10(12):M111.012500.

56. Hauser R, Ceol A, Rajagopala SV, Mosca R, Siszler G, Wermke N, et al. A second-generation protein-protein interaction network of Helicobacter pylori. Mol Cell Proteomics. 2014;13(5):1318-29.

57. Rajagopala SV, Sikorski P, Kumar A, Mosca R, Vlasblom J, Arnold R, et al. The binary protein-protein interaction landscape of Escherichia coli. Nat Biotechnol. 2014;32(3):285-90.

58. Yu H, Braun P, Yildirim MA, Lemmens I, Venkatesan K, Sahalie J, et al. High-quality binary protein interaction map of the yeast interactome network. Science. 2008;322(5898):104-10.

59. Dyer MD, Neff C, Dufford M, Rivera CG, Shattuck D, Bassaganya-Riera J, et al. The human-bacterial pathogen protein interaction networks of Bacillus anthracis, Francisella tularensis, and Yersinia pestis. PLoS One. 2010;5(8):e12089.

60. Yang H, Ke Y, Wang J, Tan Y, Myeni SK, Li D, et al. Insight into bacterial virulence mechanisms against host immune response via the Yersinia pestis-human protein-protein interaction network. Infect Immun. 2011;79(11):4413-24.

61. Park SY, Guo X. Adaptor protein complexes and intracellular transport. Biosci Rep. 2014;34(4), e00123.

62. Cohn MA, Kowal P, Yang K, Haas W, Huang TT, Gygi SP, et al. A UAF1-containing multisubunit protein complex regulates the Fanconi anemia pathway. Mol Cell. 2007;28(5):786-97.

63. Ying $H$, Zheng $H$, Scott $K$, Wiedemeyer $R$, Yan $H$, Lim $C$, et al. Mig-6 controls EGFR trafficking and suppresses gliomagenesis. Proc Natl Acad Sci U S A. 2010:107(15):6912-7.

64. Tai AW, Bojjireddy N, Balla T. A homogeneous and nonisotopic assay for phosphatidylinositol 4-kinases. Anal Biochem. 2011:417(1):97-102.

65. Dankova V, Balonova L, Straskova A, Spidlova P, Putzova D, Kijek T, et al. Characterization of tetratricopeptide repeat-like proteins in Francisella tularensis and identification of a novel locus required for virulence. Infect Immun. 2014;82(12):5035-48.

66. Nguyen G, Delarue F, Burckle C, Bouzhir L, Giller T, Sraer JD. Pivotal role of the renin/prorenin receptor in angiotensin II production and cellular responses to renin. J Clin Invest. 2002:109(11):1417-27.

67. Golovliov I, Ericsson M, Sandstrom G, Tarnvik A, Sjostedt A. Identification of proteins of Francisella tularensis induced during growth in macrophages and cloning of the gene encoding a prominently induced 23-kilodalton protein. Infect Immun. 1997:65(6):2183-9.

68. Lai XH, Golovliov I, Sjostedt A. Expression of IglC is necessary for intracellular growth and induction of apoptosis in murine macrophages by Francisella tularensis. Microb Pathog. 2004;37(5):225-30.

69. Telepnev M, Golovliov I, Sjostedt A. Francisella tularensis LVS initially activates but subsequently down-regulates intracellular signaling and cytokine secretion in mouse monocytic and human peripheral blood mononuclear cells. Microb Pathog. 2005;38(5-6):239-47.

70. Parsa KV, Ganesan LP, Rajaram MV, Gavrilin MA, Balagopal A, Mohapatra NP, et al. Macrophage pro-inflammatory response to Francisella novicida infection is regulated by SHIP. PLOS Pathog. 2006;2(7), e71.

71. Bakshi CS, Malik M, Regan K, Melendez JA, Metzger DW, Pavlov VM, et al. Superoxide dismutase B gene (sodB)-deficient mutants of Francisella tularensis demonstrate hypersensitivity to oxidative stress and attenuated virulence. J Bacteriol. 2006;188(17):6443-8.

72. Jung H, Kim MJ, Kim DO, Kim WS, Yoon SJ, Park YJ, et al. TXNIP maintains the hematopoietic cell pool by switching the function of p53 under oxidative stress. Cell Metab. 2013;18(1):75-85.

73. Roy CR, Salcedo SP, Gorvel JP. Pathogen-endoplasmic-reticulum interactions: in through the out door. Nat Rev Immunol. 2006;6(2):136-47.

74. Bhavsar AP, Guttman JA, Finlay BB. Manipulation of host-cell pathways by bacterial pathogens. Nature. 2007;449(7164):827-34. 
75. Cui J, Shao F. Biochemistry and cell signaling taught by bacterial effectors. Trends Biochem Sci. 2011;36(10):532-40.

76. Lamkanfi M, Dixit VM. Manipulation of host cell death pathways during microbial infections. Cell Host Microbe. 2010;8(1):44-54

77. Chang Q, Harter TM, Rikimaru LT, Petrash JM. Aldo-keto reductases as modulators of stress response. Chem-Biol Interact. 2003;143-144:325-32.

78. Magrane M, UniProt Consortium. UniProt Knowledgebase: a hub of integrated protein data. Database. 2011;2011:bar009.

Submit your next manuscript to BioMed Central and we will help you at every step:

- We accept pre-submission inquiries

- Our selector tool helps you to find the most relevant journal

- We provide round the clock customer support

- Convenient online submission

- Thorough peer review

- Inclusion in PubMed and all major indexing services

- Maximum visibility for your research

Submit your manuscript at www.biomedcentral.com/submit 